\title{
A equoterapia como recurso terapêutico no equilíbrio do idoso
}

\author{
Michele Marinho da Silveira*, Lia Mara Wibelinger*
}

\section{Resumo}

A equoterapia é uma técnica de reabilitação e educação que utiliza a equitação e as atividades equestres para proporcionar ao praticante benefícios físicos, psicológicos, educacionais e sociais. A atividade exige a participação do corpo inteiro, trabalhando o praticante de forma global e utilizando o cavalo como método terapêutico. Contribui, assim, para o desenvolvimento do equilíbrio, tônus, força muscular, a conscientização do próprio corpo, o aperfeiçoamento de coordenação motora, atenção, autoconfiança e autoestima. O presente estudo baseou-se em uma revisão de literatura com o objetivo de verificar os efeitos da equoterapia no equilíbrio do idoso. No idoso a equoterapia utiliza o cavalo como mediador para suprir o défice de equilíbrio em razão do seu movimento tridimensional, rítmico e balançante, que estimula o sistema vestibular, a melhora da consciência espaçotemporal, a concentração, o equilíbrio e a consolidação da segurança gravitacional, deixando o idoso menos vulnerável às quedas e as incapacidades funcionais.

Palavras-chave: Equoterapia. Equilíbrio. Idoso.

\section{Introdução}

De acordo com a Associação Nacional de Equoterapia (ANDE-Brasil, 1999), a equoterapia é um método terapêutico e educacional que utiliza o cavalo numa abordagem interdisciplinar, nas áreas da saúde, educação e equitação, buscando o desenvolvimento biopsciossocial de pessoas portadoras ou não de deficiência ou necessidades especiais.

$\mathrm{O}$ indivíduo submetido ao tratamento aprende padrões de movimentos coordenados de controle de postura para manter seu centro gravitacional sobre a base dinâmica de suporte que é criado

* Fisioterapeuta graduada pela Universidade de Passo Fundo. Especialista em Ortopedia e Traumatologia pelo Colégio Brasileiro de Estudos Sistêmicos. Mestranda em Envelhecimento Humano pela Universidade de Passo Fundo. Endereço para correspondência: Rua Palmeira, 20, apt. 803 Bairro Vera Cruz, CEP 99040460, Passo Fundo - RS. E-mail: mm.silveira@yahoo.com.br.

** Fisioterapeuta graduada pela Universidade de Santa Cruz do Sul. Professora da Faculdade de Educação Física e Fisioterapia da Universidade de Passo Fundo. Mestra e doutoranda em Gerontologia Biomédica na Pontifícia Universidade Católica do Rio Grande do Sul.

$\longrightarrow$ Recebido em agosto de 2009 - Avaliado em março de 2010.

$\hookrightarrow$ doi:10.5335/rbceh.2010.014 
pelo movimento do cavalo. Assim, ele se transforma num participante ativo no processo de terapia. (FREIRE, 1999).

O equilíbrio é um processo complexo, que depende da integração da visão, da sensação vestibular e periférica, dos comandos centrais e respostas neuromusculares e, particularmente, da força muscular e do tempo de reação. Um declínio da função relacionado à idade pode ser demonstrado em todas as partes desses sistemas, tendo como resultado o fato de que um terço da população acima de 65 anos sofre quedas a cada ano. Para obter um melhor equilíbrio o indivíduo procura manter o seu centro de massa corporal dentro dos seus limites de estabilidade, que é determinada pela habilidade em controlar a postura sem alterar a base de suporte. (OVERSTALL, 2003).

Tortora (2003) relata que existem dois tipos de equilíbrio: o equilíbrio estático refere-se ao equilíbrio e à postura do corpo (principalmente a cabeça) quando o corpo não está em movimento; o equilíbrio dinâmico refere-se ao equilíbrio e à postura do corpo (principalmente da cabeça) em resposta aos movimentos em rotação.

Paixão e Hechamnn (2002) afirmam que com o envelhecimento ocorrem perdas no sistema vestibular, nas células ciliares dos canais semicirculares, em torno de $40 \%$ depois dos setenta anos. Portanto, a diminuição da sensibilidade cutânea, da propriocepção, da acuidade visual e da sensibilidade de contraste favorece o risco de quedas, visto que as funções sensoriais estão alteradas e 0 equilíbrio, diminuído.
O presente estudo baseou-se em uma revisão de literatura que teve como objetivo verificar os efeitos da equoterapia no equilíbrio dos indivíduos idosos.

\section{Equoterapia}

Santos (2002) relata que a equoterapia é uma área nova no país e deve se dedicar à investigação científica, para que se comprovem cientificamente seus benefícios e se possa tratar as pessoas de forma inovadora e prazerosa, não se limitando somente a atendimentos em clínicas e salas fechadas, onde muitas vezes o paciente se sente desanimado a continuar com o tratamento.

Wlater e Vendramini (2000), por sua vez, enfatizam que a equoterapia emprega as técnicas de equitação e atividades equestres para proporcionar ao praticante benefícios físicos, psicológicos, educacionais e sociais. Essa atividade exige a participação de corpo inteiro, contribuindo, assim, para o desenvolvimento do tônus e da força muscular, o relaxamento, a conscientização do próprio corpo, o equilíbrio, o aperfeiçoamento da coordenação motora, a atenção, a autoconfiança e a autoestima. É um método de reabilitação e educação que trabalha o praticante de forma global.

Para Citterio (1999), a equoterapia pode ser considerada um conjunto de técnicas reeducativas que agem para superar danos sensoriais, motores, cognitivos e comportamentais, por meio de uma atividade lúdico-desportiva que tem como meio o cavalo.

De acordo com Uzun (2005), a equoterapia tem três programas de atuação: 
dependência ou hipoterapia, que diz respeito à situação na qual o praticante ainda não tem condições físicas e/ou mentais para governar o cavalo e necessita de auxiliar-guia para controlar o animal e auxiliares laterais para o apoio; semiautonomia ou reabilitação-reeducação, que acontece quando o praticante tem condições de exercer alguma atuação sobre o cavalo, geralmente montando e apeando sem auxílio; autonomia ou pré-esportiva, em que o praticante tem boas condições para atuar sobre o cavalo, participa de pequenos exercícios de hipismo e está preparado para sua reinserção social.

Conforme a ANDE-Brasil (1999), a primeira manifestação quando um ser humano está a cavalo é o ajuste tônico. Na verdade, o cavalo nunca está totalmente parado. A troca de apoio das patas, o deslocamento da cabeça ao olhar para os lados, as flexões da coluna, o abaixar e alongar do pescoço, etc. obrigam o cavaleiro a um ajuste no seu comportamento muscular, a fim de responder aos desequilíbrios provocados por esses movimentos.

Segundo Silva (2004), para que possamos entender melhor como a equoterapia funciona como método terapêutico, como se processa sua intervenção nos praticantes e por que ocorrem as melhoras em nível psicológico, cognitivo e motor, vamos expor os fundamentos teóricos nos quais encontramos bases para esse entendimento.

A ANDE-Brasil (1999) refere que a característica mais importante para a equoterapia é que o passo produz no cavalo e transmite ao cavaleiro uma série de movimentos sequenciados e simultâneos, os quais têm como resultado um movimento tridimensional, que se traduz no plano vertical em um movimento para cima e para baixo e, no plano horizontal, em um movimento para a direita e para a esquerda, segundo o eixo transversal do cavalo, um movimento para frente e para trás, segundo o eixo longitudinal. Esse movimento é completado com uma pequena torção da pelve do cavaleiro, provocada pelas inflexões laterais do dorso do animal.

Uzun (2005) descreve que a fisioterapia na equoterapia tem como finalidade proporcionar ao praticante a prevenção e o tratamento de patologias, bem como a reabilitação e o desenvolvimento de seu estado atual por meio do uso do cavalo, principalmente do movimento tridimensional e multidirecional. Cabe ao fisioterapeuta o posicionamento do praticante no cavalo, de acordo com os objetivos de estimulação, bem como a escolha de acessórios para auxiliar na montaria e nos cuidados com o transporte do praticante para o cavalo e para o solo.

Para Silva (2004), o trabalho de uma equipe interdisciplinar na equoterapia é primordial, pois esta avalia cada caso, estabelece metas e o melhor caminho para alcançá-las. A equipe deverá ser composta, no mínimo, por um adestrador de cavalo, um psicólogo e um fisioterapeuta. Observa-se também que há necessidade de que outros profissionais, como terapeuta ocupacional, fonoaudiólogo e assistente social, façam parte desta equipe.

De acordo com Freire (1999), os objetivos da equoterapia são melhorar pa- 
drões anormais pela quebra de padrões patológicos; melhorar o conhecimento do esquema corporal; melhorar a postura como um todo, normalizando o tônus corporal; estimular o equilíbrio; melhorar a coordenação espaçotemporal; educar o sistema nervoso sensorial, propriocepção e exterocepção; manter articulações íntegras e dentro da normalidade; realizar reeducação respiratória; introduzir movimentos e posturas inibidores dos reflexos; relaxar; desenvolver motivação, autoconfiança e autovalorização, que são de extrema importância para o sucesso dos objetivos citados.

Segundo a American Hippotherapy Association (NARHA, 1999), as indicações gerais para a prática da equoterapia são: crianças e adultos com disfunções neuromusculoesqueléticas de leves a severas; alteração de tônus muscular; distúrbios do equilíbrio; coordenação diminuída; comunicação inadequada; função sensório-motora alterada; assimetria postural; controle postural insuficiente; diminuição da mobilidade corporal; diminuição da atenção; distúrbios do comportamento.

Assim, a associação do ritmo com o deslocamento pélvico durante o passo do cavalo é um excelente exercício para melhorar o equilíbrio. Para Uzun (2005), o sistema vestibular é o órgão do equilíbrio, que estabelece a correlação central do movimento e da percepção. A estimulação do equilíbrio por meio do cavalo seguindo padrões do movimento tipicamente humano é única e torna-se o fundamento da equoterapia. Andar a cavalo significa, a todo instante, estimular o equilíbrio corpóreo. $\mathrm{O}$ simples fato de estar montado nos leva a minuciosos mo- vimentos de cabeça, patas e respiração do cavalo, solicitando-nos o equilíbrio.

\section{Equilíbrio}

Conforme Carnaval (1995), o equilíbrio é a habilidade que permite ao indivíduo manter o sistema musculoesquelético em uma posição estática eficaz e controlar uma postura eficiente quando em movimento, e os principais fatores que influenciam no equilíbrio são tônus muscular, funcionamento das estruturas do ouvido interno (canais semicirculares), percepção visual, sistema nervoso central.

Douglas (1999) define equilíbrio com uma situação na qual o corpo adota uma determinada posição em relação ao espaço. É um processo muito dinâmico, que luta permanentemente contra a atração gravitacional. Segundo Pinheiro (1999), o equilíbrio é o conjunto de reações de endireitamento (retificação) e inclui reações de controle cervical nos membros superiores (extensão) e membros inferiores (defesa). As reações são rápidas, seletivas, requerem alto nível de integração sensório-motora, óptica e têm relação importante com a sensibilidade proprioceptiva.

Para Umphred (2004), equilíbrio é um processo complexo, que envolve a recepção e a integração dos estímulos sensoriais e o planejamento e a execução do movimento para alcançar um objetivo, requerendo a postura ereta. É a habilidade de controlar o centro de gravidade (CG) sobre a base de suporte num dado ambiente sensorial. O CG é um ponto imaginário no espaço, calculado biome- 
canicamente, das forças e momentos medidos, em que o total de todas as forças é igual a zero. Em uma pessoa normal, estando em pé, calmamente, ele está localizado bem em frente à coluna, aproximadamente no nível S2; com o movimento do corpo e seus segmentos, a localização do CG no espaço mudará constantemente.

Um bom equilíbrio requer controle postural (força e mobilidade), visão intacta, audição e propriocepção. Alerta, atenção e memória podem também ser importantes para evitar perda de equilíbrio. (CARRIÉRE, 1999).

Para Viera (2004), episódios de desequilíbrio levam o indivíduo ao chão e definem-se como uma insuficiência súbita do controle postural; pode ser considerada uma síndrome por ser um problema que envolve aspectos e consequências biológicas, psicológicas, sociais e funcionais. A queda pode ocorrer como manifestação do organismo às demandas do momento, isto é, à exigência das tarefas do dia a dia, as quais o idoso pode não estar preparado para executar; ou pode ser um sinal de fraqueza ou de outras condições clínicas que, por facilitarem a ocorrência de quedas, levam não só a fraturas como a danos físicos, imobilidade, dependência, isolamento social, insegurança e medo.

A equoterapia vai ajudar na correção da postura, pois manter o equilíbrio significa reconhecer uma atitude corporal pelo senso postural e depois reajustar sua posição. $\mathrm{O}$ cavaleiro deve coordenar seus próprios movimentos e dissociar os gestos dos braços e pernas; portanto, é conduzido a uma melhor compreensão de seu esquema corporal. Ele adquire desde um primeiro contato o domínio corporal, aprendizagem que, num primeiro momento, vai ser favorecida pelo terapeuta, num trabalho que demanda concentração. (HERZOG, 1989).

O valor dos exercícios é que ajudam as pessoas a manterem o maior vigor possível à medida que o tempo passa. Ao fazer exercícios, podemos e esperamos aumentar a qualidade de vida, diminuir o risco de quedas e manter ou melhorar a função em diversas atividades. (KAUFFMAN, 2001).

A Fisioterapia visa melhorar a capacidade do paciente para resistir às ameaças ao seu equilíbrio, aumentar a grau de segurança do ambiente, prevenir as consequências da permanência sobre o piso e restabelecer a confiança nas suas capacidades em relação às funções de equilíbrio. (PICKLES; COMPTON; COTT, 2000).

Toigo, Leal Júnior e Ávila (2008), em sua pesquisa "O uso da equoterapia como recurso terapêutico para melhora do equilíbrio estático em indivíduos da terceira idade", concluíram que indivíduos da terceira idade saudáveis apresentam alterações no equilíbrio e que a equoterapia foi capaz de melhorar esse equilíbrio estático e, conseqüentemente, diminuir a possibilidade de quedas.

Teixeira (1999) publicou "A experiência com crianças deficientes visuais em equoterapia”. Essa pesquisa foi realizada no município de Estância Velha - RS no período de seis meses, com um grupo de seis crianças portadoras de deficiência visual congênita, em equoterapia. A autora acredita que as experiências neu- 
romusculares e a consequente integração sensorial que a vivência em equitação proporcionam formam o alicerce para a boa estruturação da criança, facilitando o futuro aprendizado intelectual, e o cavalo é um excelente mediador para suprir o défice de estimulação tátilproprioceptiva e vestibular, por seu movimento tridimensional, rítmico e balançante, que estimula o sistema vestibular; melhora a consciência espaçotemporal, a concentração, o equilíbrio e consolida a segurança gravitacional.

Silva (2004), em sua pesquisa de "Equoterapia: aplicação em distúrbios do equilíbrio", aplicando a técnica em quatro deficientes visuais, pôde observar a melhora da postura do tronco e um melhor equilíbrio em todo o grupo e que os estímulos provocados pelo movimento tridimensional do cavalo e a semelhança de seu passo à marcha humana contribuíram para esses resultados. Com esse estudo exploratório pode-se concluir que a equoterapia é um método que contribui para a melhora de aspectos psicomotores e sociais de indivíduos com cegueira congênita ou adquirida e, consequentemente, melhora sua qualidade de vida, desde que seja aplicado respeitando-se o potencial e a fase de desenvolvimento em que se encontre o indivíduo.

Silveira, Wibelinger e Sartoreto (2009) publicaram o artigo "A equoterapia como recurso terapêutico no equilíbrio de deficientes visuais", relatando que a equoterapia traz grandes benefícios a portadores de deficiência visual. Os ganhos obtidos no aspecto do equilíbrio são de grande importância, porque a mobilidade do indivíduo que não possui visão depende muito desse sentido e o seu desenvolvimento necessita ser estimulado desde o nascimento, para que tenha uma melhor qualidade de vida.

\section{Envelhecimento}

Fraiman (1988) relata que envelhecer é um processo de modificação, que se desenrola tanto na área biofisiológica como na área psicossocial; é um processo básico de todo ser vivo e até da matéria.

Rodrigues e Diogo (1996) afirmam que no Brasil considera-se idoso a pessoa que tem sessenta anos ou mais. É uma fase da vida em que as pessoas tiveram muito ganhos, mas também muitas perdas, entre as quais se destaca a saúde como um dos aspectos mais afetados nos idosos.

De acordo com Netto (2002), o envelhecimento pode ser conceituado como um processo dinâmico e progressivo, no qual há alterações morfológicas, funcionais e bioquímicas, que vão alterando progressivamente o organismo, tornando-o mais suscetível às agressões intrínsecas e extrínsecas, que terminam por levá-lo à morte.

O envelhecimento é um processo de mudanças universais, que se pauta geneticamente para a espécie e para cada indivíduo e se traduz em diminuição da plasticidade comportamental, aumento da vulnerabilidade, acumulação de perdas evolutivas e aumento da probabilidade de morte. O ritmo, a duração e os efeitos desse processo comportam diferenças individuais e de grupos etá- 
rios, dependentes de eventos e natureza genético-biológica, sócio-histórica e psicológica. (NERI, 2001).

Segundo Thompson, Skinner e Piercy (2002), os sinais do envelhecimento vão aparecendo com a idade, entre os quais se incluem: branqueamento e espessamento do cabelo, perda de elasticidade e secura da pele, audição prejudicada, visão diminuída e adaptação ao escuro limitada; a fala pode se tornar restrita; a deterioração do sistema nervoso leva à danificação da homeostase; o aprendizado e a memória a curto prazo ficam prejudicados; os limiares da dor são altos e a sensibilidade a ela diminui; o sistema cardiovascular diminui quanto à eficiência; ocorre uma menor mobilidade torácica e a capacidade vital é reduzida. Por sua vez, a memória e a personalidade não diminuem muito até os 85 anos.

Ainda conforme os mesmos autores, os idosos apresentam também diminuição da elasticidade dos tecidos moles; a estatura é diminuída pelo estreitamento dos discos intervertebrais; as articulações são menos capazes de absorver pressões e tornam-se mais rígidas, e as cartilagens, menos elásticas; o poder muscular diminui, levando à desaceleração dos movimentos e à perda da coordenação; ossos podem se tornar osteoporóticos; aparecem as dificuldades com o equilíbrio, tendo como fatores causadores a a perda da propriocepção dos tecidos nas superfícies de apoio com carga e a fraqueza muscular e as alterações degenerativas nos canais semicirculares. Por isso, quedas são um problema bem importante na velhice.(THOMPSON; SKINNER; PIERCY, 2002)
Um dos principais fatores que limitam hoje a vida do idoso é o desequilíbrio. Em 80\% dos casos não pode ser atribuído a uma causa específica, mas, sim, a um comprometimento do sistema de equilíbrio como um todo. Em mais da metade dos casos o desequilíbrio tem origem entre os 65 e os 75 anos, aproximadamente, e cerca de $30 \%$ dos idosos apresenta os sintomas nesta idade. As quedas são as consequências mais perigosas do desequilíbrio e da dificuldade de locomoção, seguidas por fraturas, que deixam os idosos acamados por dias ou meses e são responsáveis por $70 \%$ das mortes acidentais em pessoas com mais de 75 anos. (BITTAR et al., 2002).

Para Ruwer, Rossi e Simon (2005), as manifestações dos distúrbios do equilíbrio corporal têm grande impacto para os idosos, podendo levá-los à redução de sua autonomia social pela predisposição a quedas e fraturas, reduzindo suas atividades de vida diária e trazendo-lhes sofrimento, imobilidade, medo de cair novamente e altos custos com o tratamento da saúde.

Já os sistemas proprioceptivos, que surgem dos receptores tendinosos e musculares, mecanoceptores articulares e baroceptores profundos nos aspectos plantares dos pés, fornecem informações sensoriais para o controle postural. Esse conjunto de sistemas fornece ao corpo informações sobre o ambiente, permitindo a orientação necessária à medida que se movimenta ou fica estático em relação às próprias partes do corpo, seu apoio e superfície do solo. Quando as informações proprioceptivas sofrem uma diminuição ou abolição, os indivíduos passam a depender exclusivamente do 
sistema visual para manter o equilíbrio, o que ocorre no caso dos idosos. (CALAISGERMAIN, 1992).

Paixão e Hechamnn (2002) indicam que as quedas e suas consequências se fazem presentes em todas as épocas da vida, porém são vistas mais explicitamente como um problema na idade avançada. São mais frequentes nesta faixa etária, além de que os idosos, ao caírem, correm mais risco de lesões. Os distúrbios da marcha e do equilíbrio são fatores de risco para problemas como quedas e perda da independência.

As quedas são causas importantes de morbidade em idosos e podem ter consequências desastrosas. Além do risco de fraturas, há perda da confiança ao caminhar em razão do temor de novas quedas, fazendo o idoso diminuir sua mobilidade. Assim, forma-se um circulo vicioso, pois com a restrição de atividades há diminuição da força muscular, enfraquecimento das pernas, levando à condição de independência, ao isolamento social e, consequentemente, à institucionalização. (NETTO, 2002).

\section{Considerações finais}

O cavalo, além das características de seu passo, oferece situações únicas, aumentando a vivacidade do praticante em exercícios como parar e andar, mudar de direção e sentido, fazer desenhos no chão (círculo, quadrado, triângulo, entre outros). Com todas essas possibilidades, a prática da equoterapia trabalha não apenas o equilíbrio corporal, mas também o emocional do praticante. Por esse método de tratamento ser um dos únicos meios terapêuticos a oferecer mo- vimentos nos três eixos (para cima e para baixo, anteroposterior e laterolateral), intensifica-se o tempo de estimulação do equilíbrio do praticante e, consequentemente, a conquista dos resultados.

A interferência da equoterapia no equilíbrio, por meio da adequação do tônus, ritmo e simetria, dá estímulo a uma postura fisiologicamente íntegra. Existe uma estimulação permanente e constante dos órgãos do autocontrole, o que leva a um aprendizado sensóriomotor, conseguido com o movimento global de todo o corpo.

Baseado na análise dos referenciais bibliográficos estudados, foi possível concluir que a equoterapia é um recurso terapêutico eficaz no treinamento do equilíbrio do idoso, pois a biomecânica do cavalo auxilia no ritmo e na continuidade de estímulos ao sistema nervoso do praticante, reeducando a postura, auxiliando na autoestima e na autoconfiança do praticante.

The hippotheraphy as a therapeutic resource in the equilibrium of elderly

\section{Abstract}

The hippotherapy is a technique of rehabilitation and education using the riding and equestrian activities to provide practicing physical benefits, psychological, educational and social. This activity requires the participation of the whole body, the practitioner working in a general way using the horse as a therapeutic method thus contributing to the development of equilibrium, tone, muscle strength, awareness of own body, the improvement of motor coordination, attention, self-confidence and self es- 
teem. This study was based on a literature review with the objective of verifying the effects of hippotherapy in the equilibrium of elder. hippotherapy in the elder will use the horse as a mediator to meet the deficit of equilibrium due to its three-dimensional motion, swinging rhythm and that will stimulate the vestibular system, the improvement of spatial-temporal awareness, concentration, equilibrium and consolidate its own security gravitational leaving the elder less vulnerable to falls, because the manifestations of disorders of body equilibrium has great impact on the elder, may lead them to reduce their social autonomy, the predisposition to falls and fractures, reducing their activities of daily living bringing suffering, immobility, fear of falling again and with the high costs of health treatment and functional disability.

Key words: Hippotherapy. Equilibrium. Elderly.

\section{Referências}

ANDE-BRASIL. Associação Nacional de Equoterapia. Curso básico em extensão em equoterapia. Resumos. ANDE-Brasil: Brasília, 1999.

BITTAR, R. S. M et al. Síndrome do desequilíbrio no idoso. Pró-Fono _ Revista de Atualização Científica, São Paulo, v. 14, n. 1, p. 119-128, jan./abr. 2002.

CALAIS-GERMAIN, B. Anatomia para o movimento. São Paulo: Manole, 1992.

CARNAVAL, P. E. Medidas e avaliação em ciências do esporte. 2. ed. Rio de Janeiro: Sprint, 1995.

CARRIÉRE, B. Bola suíça: teoria, exercícios básicos e aplicação clínica. São Paulo: Manole, 1999.

CITTERIO, D. A hipoterapia na recuperação da pessoa portadora de deficiência e as atividades pré-esportivas. In: CONGRESSO BRASILEIRO DE EQUOTERAPIA, 1, 1999,
São Paulo, Anais... São Paulo: ANDE-Brasil, 1999.

DOUGLAS, C. R. Tratado de fisiologia aplicado á ciência da saúde. 4 ed. São Paulo: Robe Editorial, 1999.

FRAIMAN, A. P. Coisas da idade. São Paulo: Hermes, 1988.

FREIRE, H. B. G. Equoterapia teoria e técnica: uma experiência com crianças autistas. São Paulo: Vetor, 1999.

HERZOG, L. A equitação psicoterápica. Trad. Luiza Guimarães. Revista Cheval-Connexion / ANDE-Brasil, Brasília, p. 15-20, 1989.

KAUFFMAN, T. L.. Manual de reabilitação geriátrica. Rio de Janeiro: Guanabara, 2001.

NARH - North American Riding For the Handicapped Association. Preocupações e contra indicações absolutas para equoterapia. Brasília: Narha, 2001.

NERI, A. L. Palavras-chave em gerontologia. Campinas: Alínea, 2001.

NETTO, M. P. Gerontologia: a velhice e o envelhecimento em visão globalozada. São Paulo: Atheneu, 2002.

OVERSTALL, P. W. The use of balance training in elderly people with falls. Eviews in Clinical Gerontology, v. 13, n. 2, p. 153-1561, 2003.

PAIXÃO, J. R. M. C.; HECHAMNN, M. Distúrbios da postura, marcha e quedas. In: FREITAS, E. V. et.al. (Org.). Tratado de geriatria e gerontologia. Rio de Janeiro: Guanabara, 2002.

PICKLES, B.; COMPTON, A.; COTT, C. Fisioterapia na terceira idade. 2. ed. São Paulo: Santos, 2000.

PINHEIRO, J. I. Relações posturais e reflexos do ser humano e suas relações com a equoterapia. In: CONGRESSO BRASILEIRO DE EQUOTERAPIA, 1, 1999, São Paulo. Anais... São Paulo: Ande-Brasil, 1999.

RODRIGUES, R. A. P.; DIOGO, M. J. D. E. Como cuidar dos idosos. Campinas: Papirus, 1996. 
RUWER, S. L.; ROSSI, A. G.; SIMON, L. F. Equilíbrio no idoso. Revista Brasileira Otorrinolaringologia, São Paulo, v. 71, n. 3, p. 298-303, 2005.

SANTOS, R. A. Aplicação das técnicas de equoterapia e os desvios posturais laterais em crianças de 8 a 12 Anos. 2002. s. f. Monografia (Graduação em Fisioterapia) - Faculdade de Fisioterapia do Centro Universitário Feevale, Novo Hamburgo, 2002.

SILVA, C. H. Equoterapia para cegos: teoria e técnica de atendimento. Campo Grande: UCDB, 2004.

SILVEIRA, M. M.; WIBELINGER, L. M; SARTORETO, E. A equoterapia como recurso terapêutico no equilíbrio de deficientes visuais. FisioBrasil, Laranjeiras, a. 12, n. 95, p. 20-24, jun. 2009.

TEIXEIRA, C. S. A experiência com crianças deficientes visuais em equoterapia. In: CONGRESSO BRASILEIRO DE EQUOTERAPIA, 1, 1999. São Paulo. Anais... São Paulo: Ande-Brasil, 1999.

THOMSON, A.; SKINNER, A.; PIERCY, J. Fisioterapia de Tydi. 12. ed. São Paulo: Santos, 2002.

TOIGO, T.; LEAL JÚNIOR, E. C. P.; ÁVILA, S. N. O uso da equoterapia como recurso terapêutico para melhora do equilíbrio estático em indivíduos da terceira idade. Revista Brasileira de Geriatria e Gerontologia, Rio de Janeiro, v. 11, n. 3, p. 391-403, 2008.

TORTORA, G. J. Corpo humano: fundamentos de anatomia e fisiologia. 4. ed. Porto Alegre: Artmed, 2000.

UMPHRED, D. A. Fisioterapia neurológica. 2. ed. São Paulo: Manole, 1994.

UZUN, A. L. L. Equoterapia: aplicação em distúrbios do equilíbrio. São Paulo: Vetor, 2005.

VIEIRA, E. B. Manual de gerontologia. 2. ed. Rio de Janeiro: Revinter, 2004.

WALTER, G. B.; VENDRAMINI, O. M. Equoterapia: terapia com o uso do cavalo. Minas Gerais: CPT/CEE-UFV, 2000. 